\title{
Adaptive Distance Protection for Lines Connecting Converter-Interfaced Renewable Plants
}

\author{
Subhadeep Paladhi, Student Member, IEEE and Ashok Kumar Pradhan, Senior Member, IEEE
}

\begin{abstract}
Fault ride through compliance as imposed by grid codes prevents undesirable disconnection of renewable plants from the network even during fault. Diversified control schemes adopted in the converters associated with such plants modulate the voltage and current output significantly during a fault. This varies the fault characteristics of the renewable plant at times and thereby affects the performance of the distance relay protecting lines connected to such plants. In this paper, a distance protection method using local data is proposed for transmission lines connecting renewable plants. The proposed method calculates the phase angle of faulted loop current by determining the pure-fault impedance of the renewable plant at every instant following fault detection, irrespective of the control scheme associated with the plant. Utilizing the information, it calculates the line impedance up to fault point accurately. Performance of the proposed adaptive protection method is tested on renewable integrated modified 39-bus New England system using PSCAD/EMTDC simulated data and found to be accurate. Comparative assessment with the conventional distance relaying technique reveals its superiority.
\end{abstract}

Index Terms-Renewable energy power plants, distance protection, adaptive relaying, fault current modulation.

\section{NOMENCLATURE}

\section{A. Abbreviations:}

CIRP Converter-Interfaced Renewable Plant

SIR Source Impedance Ratio

PV Photovoltaic

NA-GC North American Grid Code

EU-GC European Union Grid Code

\section{B. Variables:}

$\begin{array}{ll}V_{d c} & \text { DC bus voltage of inverter } \\ I_{d c} & \text { DC bus current of inverter } \\ L_{f}, C_{f} & \text { Filter parameters (inductance and capacitance) } \\ V_{r} & \text { Operating voltage at bus } \mathrm{M} \\ I_{r} & \text { Operating current at bus } \mathrm{M} \\ Z_{a p p} & \text { Apparent impedance calculated by relay } \\ x & \text { Per unit fault distance } \\ Z_{L} & \text { Line impedance } \\ \theta_{L} & \text { Line impedance angle } \\ Z_{M F} & \text { Line impedance up to fault point from bus } \mathrm{M} \\ R_{F} & \text { Fault resistance } \\ I_{F} & \text { Current in the faulted loop } \\ \Delta Z & \text { Correction impedance } \\ E_{G} & \text { Equivalent internal voltage of grid } \\ Z_{G} & \text { Equivalent impedance of grid } \\ Z_{T r} & \text { Equivalent impedance of solar plant transformer }\end{array}$

The work is supported in part by the Central Power Research Institute, Bangalore, India, under Grant RSOP/2019/TR/07.

The authors are with the Department of Electrical Engineering, Indian Institute of Technology, Kharagpur 721302, India (e-mail: paladhisubha_ee@iitkgp.ac.in; akpradhan@ee.iitkgp.ac.in).

\author{
$E_{P V} \quad$ Equivalent internal voltage of solar plant \\ $Z_{P V} \quad$ Equivalent impedance of $\mathrm{PV}$ units with converters \\ $Z_{S P} \quad$ Equivalent impedance of Solar plant \\ $\alpha \quad$ Deviation angle \\ $\Delta I \quad$ Incremental current \\ $E_{F} \quad$ Pure-fault excitation voltage
}

C. Subscripts:
$(\mathrm{d}, \mathrm{q})$
(d, q) - axis component of the signal
(A, B, C, G) Phase-A, Phase-B, Phase-C, ground
(M, N, F) Measurements at (bus M, bus N, fault point)
$(1,2,0) \quad$ (Positive, negative, zero) sequence components

D. Superscripts:
$*$
$($ pre $, f, p f) \quad$ Prefault, during fault, pure-fault
(act, conv, prop) Actual, conventional, proposed

\section{INTRODUCTION}

L ARGE-scale converter-interfaced renewable plants (CIRPs) are of interest today to meet the increasing power demand and mitigating environmental degradation [1], [2]. Variability associated with such a renewable energy source causes power fluctuation in the plant. Maximum power point tracking control strategy is used to extract optimum power from such a plant. To satisfy the requirements of utility grid code, different control schemes are adopted in such plants [3]. Current limiting control ensures the safety of the power electronic components used in the converters during fault. Fault ride through schemes prevent the disconnection of renewable plants during network fault [4], [5]. The control schemes used in the converters modulate the voltage and current output of the plant during fault [6]. This affects the performance of distance relay protecting transmission line emanating from a renewable plant and leads to its maloperation [7]-[10].

The influence of intermittency and the control schemes associated with a CIRP on distance protection is analyzed in [8]-[11]. Different techniques are proposed for improvement of relay performance in such a situation. An improved distance relaying method is proposed in [9] for lines connecting CIRPs. The method provides a delayed decision for the relay at renewable side and also depends on the correct operation of the relay at remote end. Adaptive distance relay setting techniques are proposed in [10], [11] for protecting lines connected to wind farm. The performance of these techniques are verified for different penetration levels of the wind farm and also for wind speed variation. These techniques require information on number of participating units and wind speed 
data obtained through communication link, resulting delay in protection. These schemes are not evaluated considering embeded converter control. Improved communication assisted tripping schemes are proposed in [12], [13] for the lines emanating from CIRP. These techniques have also latency issue associated with protection decision and there is no solution in case of communication failure.

Limitation in fault current magnitude along with phase angle modulation in the presence of CIRP affects the performance of conventional distance relay protecting lines emanating from such plants, particularly for faults with significant fault resistance. To compensate the effect of fault resistance in the presence of remote infeed, adaptive distance relaying techniques have been proposed [14]-[16]. These approaches either neglect the equivalent source impedance of each end of a protected line or consider to be homogeneous to the line impedance, which is not true for a network integrating renewable energy power plants. A control based solution is provided in [7], which regulates the fault current angle from CIRP imitating the characteristics of synchronous generator. Such a technique is difficult to generalize for different types of renewable plants with various control schemes.

The converter control schemes and the variability influence the equivalent impedance of a CIRP resulting variation in source impedance ratio (SIR) [10]. An adaptive zone-1 setting technique is proposed in the presence of dynamic SIR condition following structural and operational changes in power system [17]. The technique does not address the issue associated with converter interfaced generation. Some of the available distance relays apply different techniques for setting boundaries while protecting transmission lines for different source impedance ratios (SIRs) [18]-[22]. Such relays with multiple settings requirement, for different SIRs, cannot update the setting so quickly under dynamic system conditions, which is necessary for primary protection in stepped distance relaying. Further, the distance relay settings with large fault resistance coverage during high SIR situation have load encroachment issue [21].

In summary, the distance relay protecting transmission line connected to CIRP finds limitation in case of faults with significant fault resistance due to high and variable SIR situation, which is introduced by different control schemes associated with the plant. Available protection methods for such situations either neglect the non-homogeneity caused by the control operation of CIRP or require additional information using communication link resulting delay in protection. Control based solution is difficult to generalize considering numerous control schemes associated with different types of CIRP.

In this work a distance protection approach is proposed for lines connecting converter-interfaced renewable plants mitigating the issue with fault characteristic variation. The method calculates the phase angle of faulted loop current by determining the pure-fault sequence impedances of the renewable plant with every new samples of voltage and current data available to the relay. Thereby the proposed adaptive approach uses it to obtain the line impedance up to the fault point from relay bus, irrespective of the control schemes associated with different types of CIRP. The performance of the proposed method is tested on renewable integrated modified 39-bus New England system using PSCAD/EMTDC simulation data for different fault situations with variation in renewable plant capacity, its type and system conditions including power swing. The proposed method can be applied for protection of any transmission line, CIRP connected or not. Comparative assessment with conventional distance relaying technique ensures its superiority.

The paper is organized as follows: Section II introduces the renewable integrated test system used in this work with brief description on the control scheme applied for the CIRPs. The issue associated with distance relay for protecting lines connected to CIRP is analyzed in Section III. The proposed adaptive distance protection method is formulated in Section IV and the results evaluating its performance in different situations are provided in Section V. Section VI concludes the work.

\section{SySTEM DESCRIPTION}

The issue discussed in this work and the performance of the proposed solution technique for the mitigation are tested on 345 kV, 60 Hz, 39-bus New England system [23] integrating renewable plant, as shown in Fig. 1. Simulations are carried out using EMTDC/PSCAD. Voltage and current phasors are estimated using 1-cycle discrete Fourier transform with a sampling rate of $3.84 \mathrm{kHz}$.

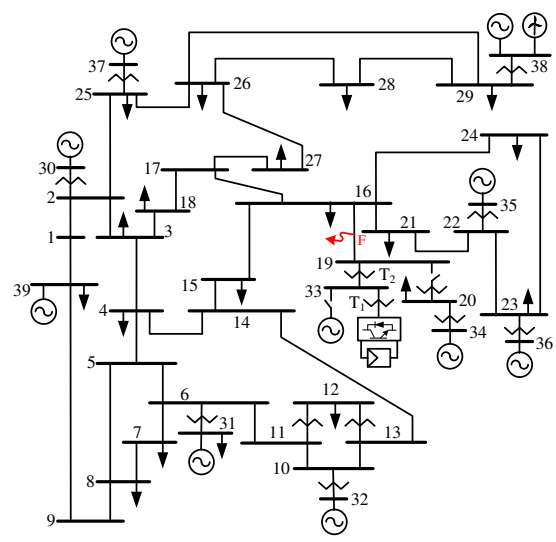

Fig. 1. CIRP integrated modified 39-bus New England system.

The generator connected at bus 33 is replaced by a 300 MW solar photovoltaic (PV) plant. The solar plant consists of multiple PV units, each connected to the common coupling bus (33) through a DC/AC inverter and transformer [24]. The solar based CIRP is controlled in synchronous reference frame, which controls the fault current along with the filter of the converter, as shown in Fig. 2 [7]. The grid side and converter side voltages are denoted by $V_{\text {grid }}$ and $V_{\text {inv }}$ respectively. Feedforward compensation is provided, as shown in Fig. 2, to decouple the converter operation from the disturbances occurred in the grid side. This maintains the voltage across the filter to be balanced even during unbalanced situation in grid side and consequently generates balanced current in output. However, a small amount of unbalance is always found to be present in output current due to the inability of PI controllers in suppressing dq current oscillations at twice the nominal frequency during asymmetrical faults [25]. 
The solar plant is modeled satisfying North American grid codes (NA-GCs) and operates close to unity power factor [26]. As to the grid code (GC), the solar plant rides through the low voltage situation during fault without imposing any regulation on active and reactive components of fault current [5], [8]. On the other hand, the European Union grid code (EU-GC) imposes high priority on reactive power support to improve the voltage profile at common coupling point [8]. The reactive current reference for the purpose is calculated as in [27]. For verification of the impact of different renewable sources, the solar plant is replaced in some cases with Type-III and Type-IV wind farms consisting of standard control schemes as in [28], [29]. All these CIRPs being connected to bulk-power system consider fault ride through and internal protection guideline as to the NERC reliability standard [30].

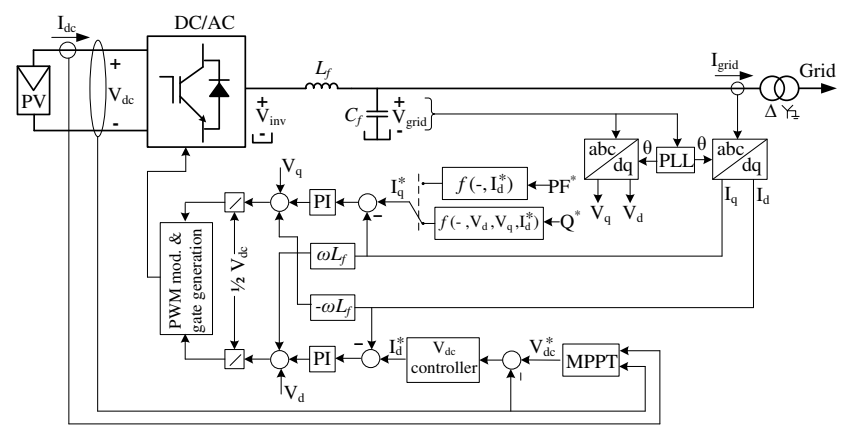

Fig. 2. Control scheme applied to the solar based CIRP.

Performance of the distance protection method is tested for the relay at bus 19 for faults in line 19-16. For emphasizing the issue in the presence of converter interfaced renewable plants, bus 20 is disconnected from bus 19, which isolates the generator at bus 34 and load at bus 20 from rest of the system. In order to maintain proper generation and load balance, bus 15, 16 and 24 loads are modified suitably. A 300 MW Type-III wind farm is connected at bus 38 with the existing synchronous generator, sharing its total generation.

\section{Issue With Distance RELAY FOR LINES CONNECTING CIRP}

Fig. 3 shows a transmission line connecting solar based CIRP. The solar plant consists of multiple solar photovoltaic units with each one connected to the collector bus through DC/AC inverter and a step-up transformer. $\mathrm{Z}_{\mathrm{L}}$ represents the impedance of line $\mathrm{MN}$ and a fault is considered at a distance of $x$ pu from bus $\mathrm{M}$ with a fault resistance, $\mathrm{R}_{\mathrm{F}}$.

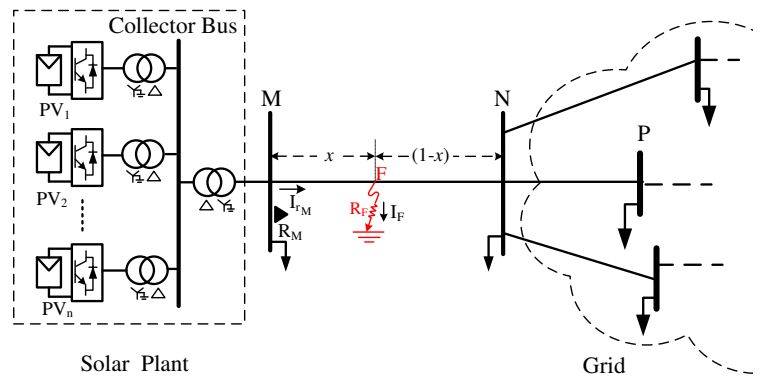

Fig. 3. Transmission network integrating solar plant to grid.
Apparent impedance $\left(Z_{a p p}\right)$ calculated by the distance relay at bus $\mathrm{M}\left(\mathrm{R}_{\mathrm{M}}\right)$ for the situation is given by [31],

$$
Z_{a p p}=\frac{V_{r_{M}}}{I_{r_{M}}}=x Z_{1 L}+\left(\frac{I_{F}}{I_{r_{M}}}\right) R_{F}=Z_{M F}+\Delta Z
$$

Where, $V_{r_{M}}$ and $I_{r_{M}}$ are the operating voltage and current for the relay, $\mathrm{R}_{\mathrm{M}}$. $I_{F}$ represents the faulted loop current. Table I provides $V_{r}, I_{r}$ and $I_{F}$ for different fault types.

TABLE I

DistancE RELAY OPERATING MEASUREMENTS AND FAULTED LOOP CURRENT FOR DIFFERENT FAULT TYPES

\begin{tabular}{c|c|c|c}
\hline \hline Fault type & $V_{r}$ & $I_{r}$ & $I_{F}$ \\
\hline $\mathrm{AG}$ & $V_{A G}$ & $I_{A}+K_{0 L} I_{0}$ & $I_{A F}$ \\
\hline $\mathrm{BG}$ & $V_{B G}$ & $I_{B}+K_{0 L} I_{0}$ & $I_{B F}$ \\
\hline $\mathrm{CG}$ & $V_{C G}$ & $I_{C}+K_{0 L} I_{0}$ & $I_{C F}$ \\
\hline $\mathrm{ABG}$ & $V_{A B}$ & $I_{A}-I_{B}$ & $I_{A F}-I_{B F}$ \\
\hline $\mathrm{BCG}$ & $V_{B C}$ & $I_{B}-I_{C}$ & $I_{B F}-I_{C F}$ \\
\hline $\mathrm{CAG}$ & $V_{C A}$ & $I_{C}-I_{A}$ & $I_{C F}-I_{A F}$ \\
\hline $\mathrm{ABC}$ & $V_{A G}$ & $I_{A}$ & $I_{1 F}$ \\
\hline
\end{tabular}

where $K_{0 L}$ is the zero sequence compensation factor for line $\mathrm{MN}$ and expressed as, $K_{0 L}=\frac{Z_{0 L}-Z_{1 L}}{Z_{1 L}} .1,2$ and 0 in subscript represent the positive, negative and zero sequence components respectively.

As in (1), apparent impedance calculated by relay $R_{M}$ includes an additional impedance, $\Delta Z\left(=\left(I_{F} / I_{r_{M}}\right) R_{F}\right)$ along with the impedance of the line up to fault point, $Z_{M F}$ $\left(=x Z_{1 L}\right)$. Fault current limitation by the CIRP interfacing converters causes the ratio, $\left(I_{F} / I_{r_{M}}\right)$ to be very high compared to a conventional power network with synchronous generator based sources. This results a large difference between $Z_{M F}$ and $Z_{a p p}$. Fault current modulation by the solar plant is regulated by the control schemes associated with the plant. This introduces non-homogeneity in the system compared to a sunchronous generator based network and results in a significant phase angle difference between $I_{r_{M}}$ and $I_{F}$ [7], [8]. Such phase angle difference adds a significant reactive part in $\Delta Z$ and deviates the $Z_{a p p}$ along the imaginary axis in $\mathrm{R}-\mathrm{X}$ plane.

The above mentioned fault current characteristics in the presence of solar based CIRP are evident from the results shown in Fig. 4, for a phase B-to-phase C-to-ground (BCG) fault in line 19-16 of the system in Fig. 1 at a distance of 0.4 pu from bus 19 with a fault resistance of $20 \Omega$. Solar plant complied by NA-GC operates close to unity power factor even during fault. This results in the fault current at solar plant side leading significantly to the current through $R_{F}$. This is evident from Fig. 4(a), where delta line current between phaseB and phase-C at bus $19\left(I_{B_{19}}-I_{C_{19}}\right)$ leads the faulted loop current $\left(I_{B_{F}}-I_{C_{F}}\right)$ by an angle of $56^{0}$. With such phase angle relationship leads the $Z_{a p p}$ to shift towards negative imaginary axis in R-X plane. Fault current limitation in solar plant results $\left(\left|I_{B_{F}}-I_{C_{F}}\right| /\left|I_{B_{19}}-I_{C_{19}}\right|\right)$ to be very high, which can be evident from Fig. 4(b). This results a large deviation in $Z_{a p p}$ from $Z_{M F}$. Effect of such large deviation in apparent impedance toward negative imaginary axis results the $Z_{a p p}$ calculated by the relay at bus 19 to settle in $4^{\text {th }}$ quadrant of R-X plane, 
as shown in Fig. 5 for a distance relay with fault resistance coverage of $100 \Omega$. This leads the fault to be unidentified in proper zone of the relay setting. Fault current from solar plant being regulated by the associated control schemes gets modulated differently with the fault situation and solar plant operation status. This results significant difference in $Z_{a p p}$ calculated by the relay, even for faults at same location with equal fault resistance. Thus there is a need for new protection technique, adaptive to the fault current modulation in the presence of CIRP to prevent such distance relay maloperation.

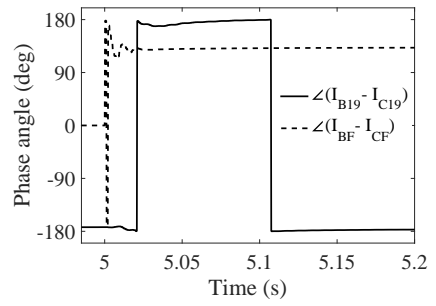

(a)

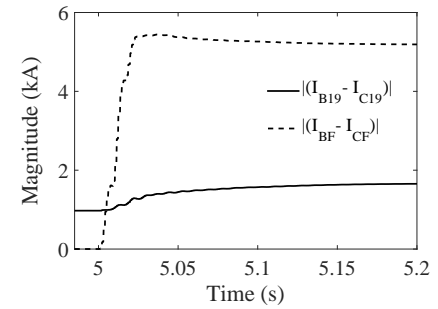

(b)
Fig. 4. Fault current characteristics showing (a) phase angle modulation and (b) fault current limitation in the presence of solar based CIRP.

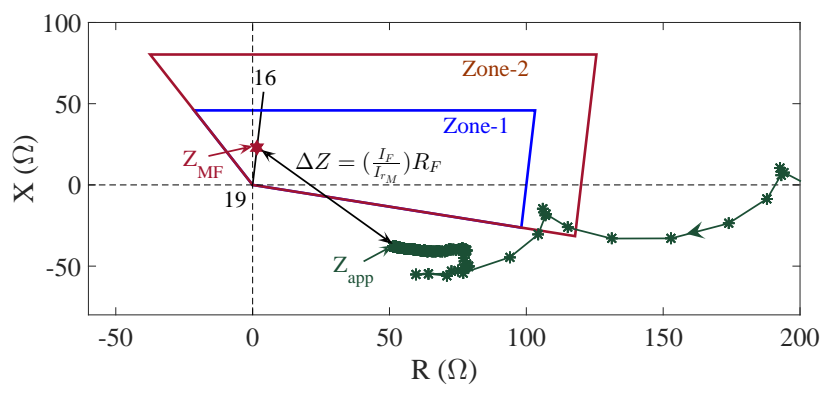

Fig. 5. Distance relay maloperation in the presence of solar based CIRP.

\section{Proposed Adaptive Relaying Method}

Fig. 6(a) represents the issue associated with distance relay in the presence of CIRP, as discussed in section II. On the other hand, renewable plant complied with EU-GC generates reactive current during fault to maintain the voltage level at the connected bus. This results $I_{F}$ to lead $I_{r_{M}}$. Distance relaying for such a situation is represented in Fig. 6(b). $\theta_{1 L}$ represents the phase angle associated with $Z_{1 L} . R_{a p p}$ and $X_{a p p}$ are the resistance and reactance associated with $Z_{a p p}$ calculated by the relay.

Using the geometric property, the deviation angle $(\alpha)$ in Fig. 6 can be expressed as,

$$
\alpha=\tan ^{-1}\left(\frac{\left|Z_{M F}\right| \sin \theta_{1 L}-X_{a p p}}{\left|Z_{M F}\right| \cos \theta_{1 L}-R_{a p p}}\right)
$$

$Z_{M F}$, with an angle same as the line impedance angle, can be obtained from (2) as follows,

$$
Z_{M F}=\frac{X_{a p p}-R_{a p p} \tan \alpha}{\sin \theta_{1 L}-\cos \theta_{1 L} \tan \alpha}\left(\cos \theta_{1 L}+j \sin \theta_{1 L}\right)
$$

$\alpha$ in Fig. 6 is the gradient of $\Delta Z$ and can be expressed as,

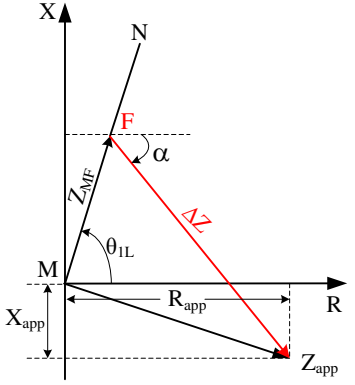

(a)

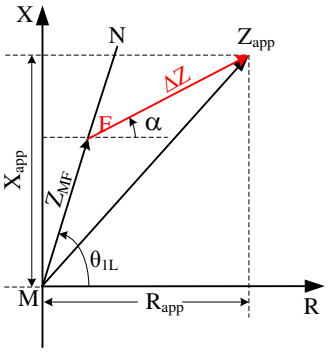

(b)
Fig. 6. Impedance diagram of distance relaying with CIRP supplying fault current (a) leading and (b) lagging to the current in the faulted loop.

$$
\alpha=\arg \left(\frac{I_{F}}{I_{r_{M}}} R_{F}\right)=\arg \left(\frac{I_{F}}{I_{r_{M}}}\right)
$$

$\alpha$ should be calculated correctly to obtain $Z_{M F}$ using (3). As in (4), $\alpha$ is a function of $I_{F}$, which depends on the remote end current of the protected line. Techniques to determine $\alpha$ using local data for different fault types are proposed below with a detailed analysis of the CIRP integrated power network.

Fig. 7 shows the equivalent diagram of the system of Fig. 3. The positive sequence model of conventional power grid connecting only synchronous generators is represented using a voltage source $\left(E_{G}\right)$ with an impedance $\left(Z_{G}\right)$ in series. A grid connected solar plant is considered as a voltage controlled current source. At any particular instant, a current source can be converted to an equivalent voltage source $\left(E_{P V}\right)$ with a series impedance $\left(Z_{P V}\right)$ [32]. Such a voltage source based representation is shown in Fig. 7. $Z_{P V}$ changes at each measurement instant, in accordance with the variation of solar irradiance, plant status and control operation. Such a representation satisfies the controlled voltage and current output at the terminal of CIRP [33], [34]. Equivalent impedance of the solar plant consisting of $Z_{P V}$ and transformer impedance $\left(Z_{T r}\right)$ in series is represented as $Z_{S P}$ further in the analysis.

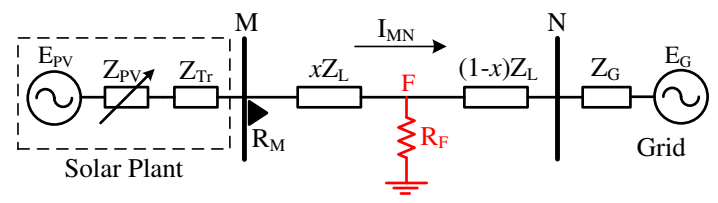

Fig. 7. Two bus equivalent model of solar plant integrated network.

Equivalent models of such a network during prefault and a three phase fault condition consist of different equivalent impedances, as shown in Fig. 8. $Z_{1 S P}^{\text {pre }}$ and $Z_{1 S P}^{f}$ represent the equivalent positive sequence impedances of solar plant during the prefault and fault, which vary with the measurement instances depending on the control scheme and system condition.

Using Kirchhoffs current law for the circuit in Fig. 8(b), the faulted loop current through $\mathrm{R}_{\mathrm{F}}$ can be expressed as,

$$
I_{1 F}=I_{1 M}^{f}+I_{1 N}^{f}
$$

where, $I_{1 M}^{f}$ and $I_{1 N}^{f}$ represent the fault currents at bus $\mathrm{M}$ and $\mathrm{N}$ respectively. 


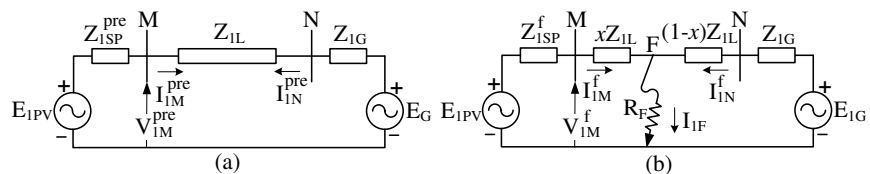

Fig. 8. Equivalent model of a solar based CIRP integrated network during (a) prefault and (b) fault situations.

Currents at both ends during prefault are equal in magnitude, but opposite in phase, as shown in Fig. 8(a), Thus (5) can be rewritten as,

$$
I_{1 F}=\Delta I_{1 M}+\Delta I_{1 N}
$$

where incremental current, $\Delta I_{1(M, N)}$ at each end is represented as in (7).

$$
\Delta I_{1(M, N)}=I_{1(M, N)}^{f}-I_{1(M, N)}^{p r e}
$$

Using the relation of (7) and by applying superimposed principle, a pure-fault sequence network for a CIRP integrated system is derived in Fig. 9 . Note that pure-fault impedance of solar plant $\left(Z_{1 S P}^{p f}\right)$ is different from $Z_{1 S P}^{\text {pre }}$ and $Z_{1 S P}^{f}$. The excitation voltage $\left(E_{F}\right)$ for the pure-fault network is the voltage at $\mathrm{F}$ during the prefault measurement instance.

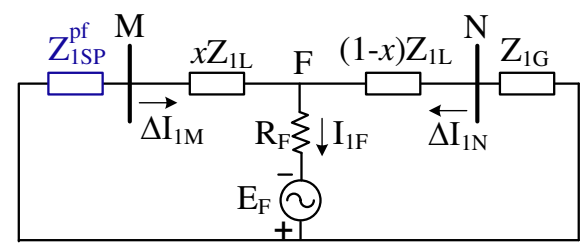

Fig. 9. Pure-fault network model of a CIRP integrated transmission network.

Applying current distribution property in Fig. 9, incremental current at bus $\mathrm{M}\left(\Delta I_{1 M}\right)$ can be expressed as,

$$
\Delta I_{1 M}=\frac{(1-x) Z_{1 L}+Z_{1 G}}{Z_{1 S P}^{p f}+Z_{1 L}+Z_{1 G}} I_{1 F}
$$

Using the relation (5) in (8), $Z_{1 S P}^{p f}$ can be expressed as,

$$
Z_{1 S P}^{p f}=\frac{\left(I_{1 M}^{f}+I_{1 N}^{f}\right)\left((1-x) Z_{1 L}+Z_{1 G}\right)}{I_{1 M}^{f}-I_{1 M}^{p r e}}-\left(Z_{1 L}+Z_{1 G}\right)
$$

Above can be simplified as,

$$
Z_{1 S P}^{p f}=\left(I_{1 M}^{p r e} Z_{M G}-I_{1 M}^{f} x Z_{1 L}+I_{1 N}^{f} Z_{F G}\right) /\left(I_{1 M}^{f}-I_{1 M}^{p r e}\right)
$$

where, $Z_{M G}=Z_{1 L}+Z_{1 G}$ and $Z_{F G}=(1-x) Z_{1 L}+Z_{1 G}$. Introducing $E_{1 G}$ of Fig. 8 , in the numerator of the above equation, we get

$$
Z_{1 S P}^{p f}=\frac{\left(E_{1 G}+I_{1 M}^{p r e} Z_{M G}\right)-\left(E_{1 G}-I_{1 N}^{f} Z_{F G}+I_{1 M}^{f} x Z_{1 L}\right)}{I_{1 M}^{f}-I_{1 M}^{p r e}}
$$

With the equivalent networks during prefault and fault, as shown in Fig. 8, the two parts of the numerator in (11) can be simplified as,

$$
\begin{aligned}
V_{1 M}^{p r e} & =E_{1 G}+I_{1 M}^{p r e} Z_{M G} \\
V_{1 M}^{f} & =E_{1 G}-I_{1 N}^{f} Z_{F G}+I_{1 M}^{f} x Z_{1 L}
\end{aligned}
$$

Using the relation (12) in (11), the pure-fault impedance becomes,

$$
Z_{1 S P}^{p f}=\left(V_{1 M}^{p r e}-V_{1 M}^{f}\right) /\left(I_{1 M}^{f}-I_{1 M}^{p r e}\right)
$$

With the concept as derived above, the pure-fault sequence diagrams of the system in Fig. 7 are shown in Fig. 10 for AG, $\mathrm{BC}$ and BCG faults representing all three asymmetrical fault types. $R_{p h}$ represents the phase-to-phase arcing resistance [8]. $\mathrm{dYg}$ connection of the main transformer connecting solar plant results in zero sequence current at bus $M$ to flow through a constant impedance, as shown in Fig. 10. Solar plant controlled in synchronous reference frame and with feedforward compensation is set to generate balanced current output, as described in Section II. Still, there always exist a small negative sequence current, especially during the transient period (up to 1.5 cycles following fault inception) when the relays providing primary protection usually take the decision [35], [36]. Thus, the small uncontrolled negative sequence current from solar plant makes $Z_{2 S P}^{p f}$ to be very high and variable with time and fault situation. Some of the available standards reveal that the CIRP interfacing inverters are being configured to be capable of providing negative sequence current [30]. Equivalent negative sequence circuit of CIRP with such an inverter configuration can also be represented with a variable impedance, as used in this work.

With the pure-fault sequence networks for different fault types, $\alpha$ in (4) can be determined as follows.

\section{A. For three phase fault}

As in (1), for a three phase fault, the relay $R_{M}$ calculates the $Z_{a p p}$ using (14).

$$
Z_{a p p}=\frac{V_{A M}}{I_{A M}}
$$

With the sequence components calculated using phase-A as reference, the $I_{r_{M}}$ for three phase fault can written as,

$$
I_{r_{M}}=I_{A M}=I_{1 M}
$$

Applying current distribution property in the pure-fault sequence network of such fault type (Fig. 9), current in the faulted loop through $\mathrm{R}_{\mathrm{F}}\left(I_{1 F}\right)$ can be expressed as,

$$
I_{1 F}=\Delta I_{1 M}\left(\frac{Z_{1 S P}^{p f}+Z_{1 L}+Z_{1 G}}{(1-x) Z_{1 L}+Z_{1 G}}\right)
$$

Homogeneity in a conventional transmission network allows the grid equivalent impedance $\left(Z_{G}\right)$ to be expressed in terms of protected line impedance $\left(Z_{L}\right)$ with real valued multiplier $\left(K_{1,2,0}\right)$ for each sequence component, as in (17).

$$
Z_{(1,2,0) G}=K_{(1,2,0)} Z_{(1,2,0) L}
$$

Grid strength being sufficiently large compared to solar plant, $Z_{1 G}$ is much smaller than $Z_{1 L}$ and $Z_{1 S P}^{p f}$. Thus neglecting $Z_{1 G}$ in the numerator of (16) and using (17), $I_{1 F}$ can be approximated as,

$$
I_{1 F} \approx \Delta I_{1 M}\left(\frac{Z_{1 S P}^{p f}+Z_{1 L}}{\left(1-x+K_{1}\right) Z_{1 L}}\right)
$$

Using $I_{r_{M}}$ obtained from (15) and $I_{1 F}$ obtained from (18), $\alpha$ in (4) for three phase fault is expressed as,

$$
\alpha_{A B C}=\arg \left(\frac{I_{F}}{I_{r_{M}}}\right)=\arg \left(\frac{\left(1+\frac{Z_{1 S P}^{p f}}{Z_{1 L}}\right) \Delta I_{1 M}}{I_{1 M}^{f}}\right)
$$



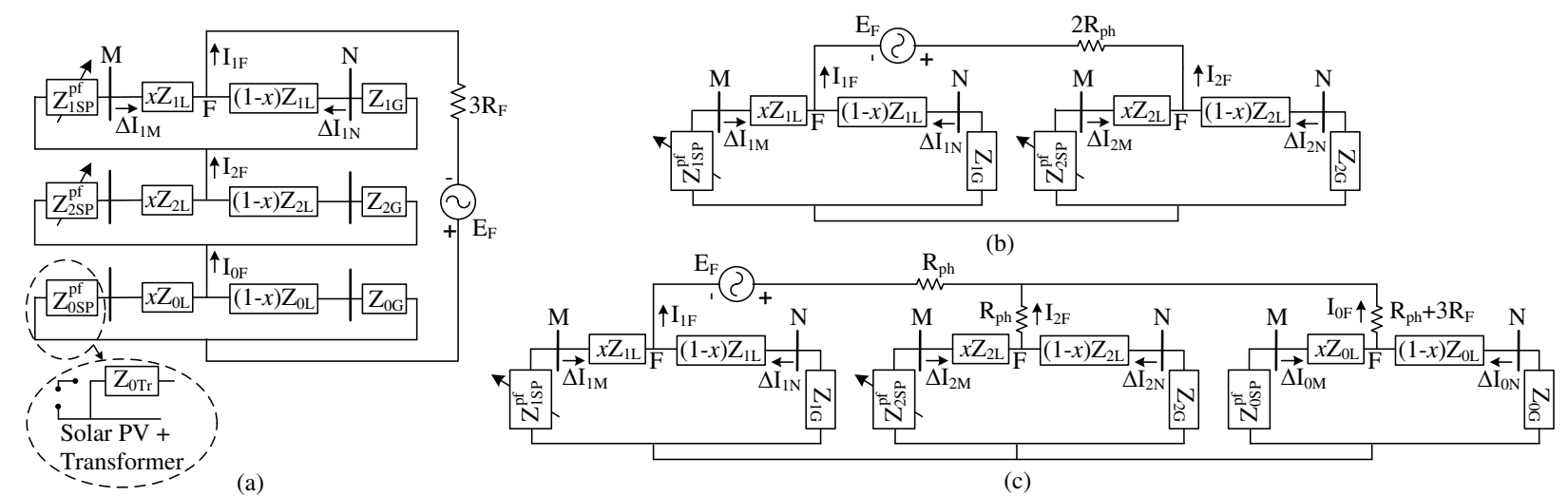

Fig. 10. Pure-fault sequence network for (a) AG, (b) BC and (c) BCG fault with solar based CIRP connected at bus M.

\section{B. For phase-A-to-ground fault}

$Z_{\text {app }}$ calculated by $\mathrm{R}_{\mathrm{M}}$ for $\mathrm{AG}$ fault is expressed as,

$$
Z_{a p p}=V_{A M} /\left(I_{A M}+K_{O L} I_{0 M}\right)
$$

As in Fig. 10(a), current in the faulted loop is $I_{1 F}$. Distribution of $I_{1 F}$ in Fig. 10(a) is similar as in Fig. 9. Thus, using $I_{1 F}$ and $I_{r_{M}}$ obtained from (18) and (20) respectively, $\alpha$ for AG fault can be expressed as,

$$
\alpha_{A G}=\arg \left(\frac{I_{F}}{I_{r_{M}}}\right)=\arg \left(\frac{\left(1+\frac{Z_{1 S P}^{p f}}{Z_{1 L}}\right) \Delta I_{1 M}}{I_{A M}^{f}+K_{0 L} I_{0 M}^{f}}\right)
$$

\section{For phase-B-to-phase-C fault}

Using sequence components, $Z_{a p p}$ calculated in (1) for BC fault can be expressed using (22) [37].

$$
Z_{\text {app }}=\frac{V_{B C_{M}}}{I_{B C_{M}}}=\frac{V_{1 M}-V_{2 M}}{I_{1 M}-I_{2 M}}
$$

From Fig. 10(b), the relation between the sequence currents in the faulted loop can be written as,

$$
I_{1 F}=-I_{2 F}
$$

Thus, the current in the faulted loop is expressed as,

$$
I_{F}=I_{1 F}-I_{2 F}=2 I_{1 F}
$$

Using current distribution of $I_{1 F}$ as in (18), $\alpha$ for BC fault can be expressed as,

$$
\alpha_{B C}=\arg \left(\frac{I_{F}}{I_{r_{M}}}\right)=\arg \left(\frac{\left(1+\frac{Z_{1 S P}^{p f}}{Z_{1 L}}\right) \Delta I_{1 M}}{I_{1 M}^{f}-I_{2 M}^{f}}\right)
$$

\section{For phase-B-to-phase-C-to-ground fault}

For BCG fault, $\mathrm{R}_{\mathrm{M}}$ calculates $Z_{a p p}$ using (22), similar to BC fault. From the faulted loop consisting of positive and negative sequence network components at solar plant side in Fig. 10.c, the loop current can be expressed as,

$$
I_{F}=I_{1 F}-I_{2 F}
$$

Using current distribution property for $I_{2 F}$ in Fig. 10.c similar to (18) for $I_{1 F}, \alpha$ for BCG fault can be determined using (27).

$$
\alpha_{B C G}=\arg \left(\frac{\left(1+\frac{Z_{1 S P}^{p f}}{Z_{1 L}}\right) \Delta I_{1 M^{-}}\left(1+\frac{Z_{2 S P}^{p f}}{Z_{2 L}}\right) \Delta I_{2 M}}{I_{1 M}^{f}-I_{2 M}^{f}}\right)
$$

where, $Z_{2 S P}^{p f}$ is the negative sequence pure-fault impedance of solar plant. With a balanced prefault condition, this can be determined as follows,

$$
Z_{2 S P}^{p f}=-\frac{\Delta V_{2 M}}{\Delta I_{2 M}}=-\frac{V_{2 M}^{f}}{I_{2 M}^{f}}
$$

In an ideal condition, a solar plant with voltage feedforward compensation generates balanced current, even during asymmetrical fault. In order to avoid any compromise with the sensitivity of a protection scheme in the presence of such negligible negative sequence current, the negative sequence circuit of the solar plant can be represented with an open circuit, as shown in Fig. 11. As the current distribution principle used in (27) for $I_{2 F}$ cannot be applied for such a circuit, $\alpha$ is calculated using $V_{2 M}^{f}$ as follows.

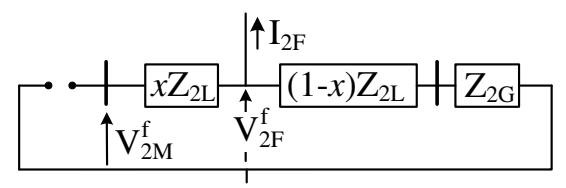

Fig. 11. Negative sequence network in case of balanced current output from CIRP during asymmetrical fault.

$V_{2 M}^{f}$ in Fig. 11 is equal to $V_{2 F}^{f}$. $I_{2 F}$ only flows through the grid side. Using the impedance homogeneity between grid and protected line as in (17), $I_{2 F}$ can be expressed as,

$$
I_{2 F}=-\frac{V_{2 F}^{f}}{(1-x) Z_{2 L}+Z_{2 G}} \approx-\frac{V_{2 M}^{f}}{\left(1-x+K_{2}\right) Z_{2 L}}
$$

Using $I_{1 F}$ and $I_{2 F}$ obtained from (18) and (29) respectively, faulted loop current for BCG fault can be expressed as,

$$
I_{F}=\left(\left(\frac{Z_{1 S P}^{p f}+Z_{1 L}}{\left(1-x+K_{1}\right) Z_{1 L}}\right) \Delta I_{1 M}+\frac{V_{2 M}^{f}}{\left(1-x+K_{2}\right) Z_{2 L}}\right)
$$


As $K_{1}=K_{2}$ for a transmission network, $\alpha$ for BCG fault (when $I_{2 M}=0$ ) can be expressed as,

$$
\alpha_{B C G}=\arg \left(\left(\left(1+\frac{Z_{1 S P p f}}{Z_{1 L}}\right) \Delta I_{1 M}+\frac{V_{2 M}^{f}}{Z_{2 L}}\right) / I_{1 M}^{f}\right)
$$

\section{E. Proposed adaptive relaying algorithm}

Steps associated with the proposed distance relaying method are shown in Fig. 12. In the presence of fault current limiting control with CIRP, the drop in voltage is prominent during fault. Following detection of the fault using voltage-drop principle, the fault type is classified using the voltage measurement available at the relay, as in [26]. According to the fault type, relay calculates the deviation angle, $\alpha$ associated with $\Delta Z$ using pure-fault sequence impedances of solar plant obtained from (13) and (28). In case of unavailability of negative sequence current from CIRP, even during asymmetrical fault, the method calculates the deviation angle using negative sequence voltage measured by the relay, as in (31) for BCG fault. This deviation angle $\alpha$ is used in (3) with the apparent impedance calculated using (1) to determine $Z_{M F}$, the line impedance up to fault point. Considering measurement errors and other uncertainties, the instantaneous tripping is provided up to $80 \%$ of the line.

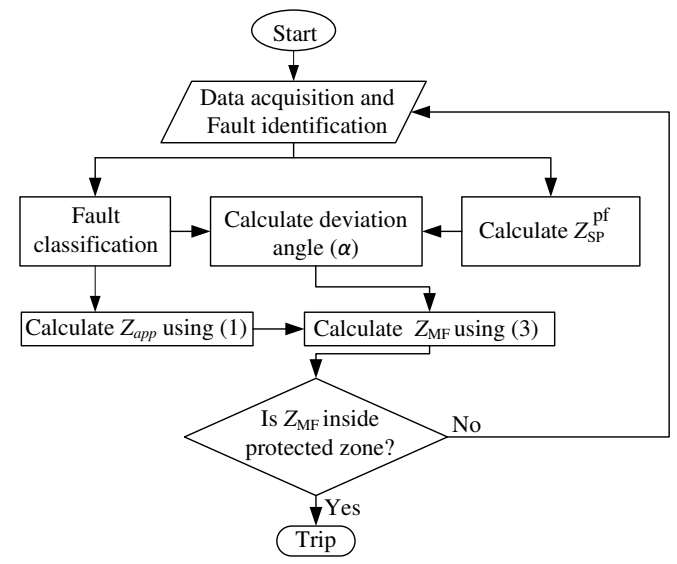

Fig. 12. Flow diagram for the proposed adaptive relaying technique.

\section{RESUlTS}

Proposed protection method is tested for CIRP integrated modified 39-bus New England system as shown in Fig. 1. Performance of relay at bus 19 protecting line 19-16 is evaluated for different fault situations considering variation in system conditions and source types. Comparative assessment with conventional distance relaying technique is provided to demonstrate the strength of the proposed method.

\section{A. Performance of the proposed method and Comparative assessment}

Fault current modulation caused by the solar plant results maloperation of conventional distance relay at bus 19 for faults in line 16-19. Fig. 13(a) shows such a maloperation scenario for a BCG fault at a distance of $0.4 p u$ from bus 19 with a fault resistance of $20 \Omega$. The proposed method calculates pure-fault sequence impedances of the solar plant with every new sample pairs of voltage and current data. Using the information, it starts calculating the line impedance up to fault point $\left(Z_{M F}^{\text {prop }}\right)$ with every apparent impedance points seen by relay $\left(Z_{a p p}^{c o n v}\right)$ following fault detection. As evident from the result, the proposed adaptive relaying method identifies the fault correctly with its accurate location. All the impedance points calculated by the proposed technique $\left(Z_{M F}^{p r o p}\right)$ corresponding to the apparent impedance points $\left(Z_{a p p}^{c o n v}\right)$ of the distance relay following fault detection are shown in Fig. 13(b). It demonstrates that the impedance points calculated following the initial transient period by the proposed technique coincide at the same impedance point on line and identifies the fault accurately within 1 cycle following fault inception.

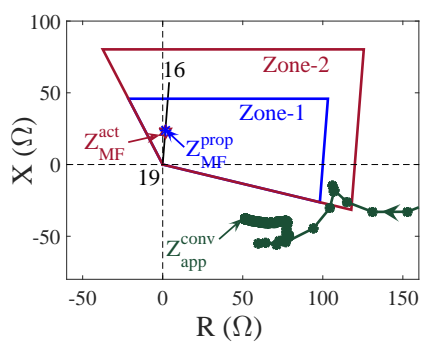

(a)

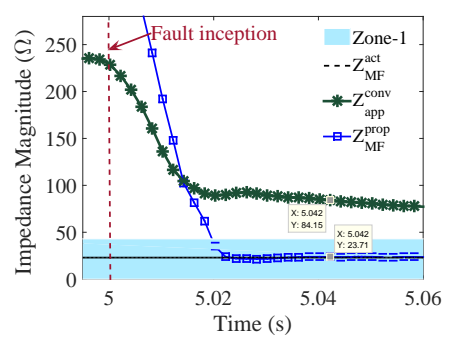

(b)
Fig. 13. Performance of the conventional distance relaying and proposed approach with (a) distance relay characteristics and (b) time of operation.

\section{B. Performance evaluation for different fault types and fault resistances and Comparative assessment}

Fault current modulation by the control schemes associated with CIRP results in significant phase angle difference between local operating current and faulted loop current. Such phase angle differences, observed for different types of faults created in line 19-16 at a distance of $0.4 \mathrm{pu}$ from bus 19 with equal fault resistance, are shown in Fig. 14. It is observed that the phase angle difference increases with the fault severity, as it is largest for $\mathrm{ABC}$ fault. Changes in control scheme and fault severity modulate these phase angle differences. As the fault resistance associated with phase faults $(\mathrm{ABC}$ and $\mathrm{BC})$ are not much high, compared to ground faults (AG and $\mathrm{BCG}$ ), relays are more prone to maloperation for these types of faults.

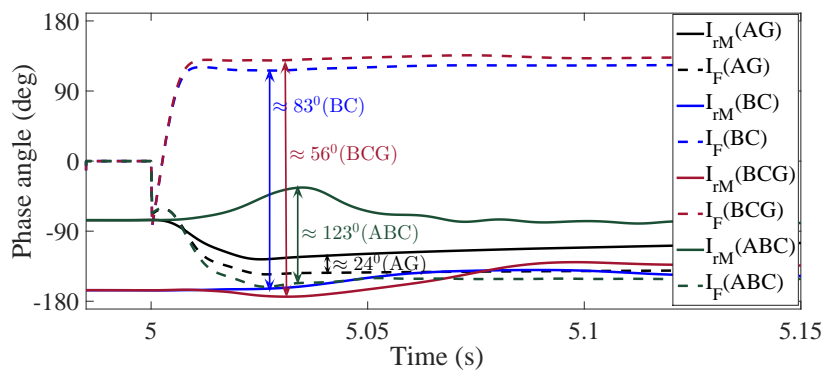

Fig. 14. Phase angle difference between operating current and faulted loop current for different fault types.

Results for different types of faults at same location in line 19-16 with large variation in fault resistances are provided in Table II. Fault resistance for ground faults (AG and BCG) is varied from $5 \Omega$ to $100 \Omega$, whereas for phase faults (BC and $\mathrm{ABC}$ ) the variation is considered up to $30 \Omega$. Results show 
that the apparent impedance seen by conventional distance relay deviates significantly from its actual value and remains unidentified by the zone-1 setting in some cases, whereas the impedance calculated by the proposed method is almost identical with the actual one in each case. Results provided for BCG fault with a $R_{F}$ of $50 \Omega$, as in the shaded row of Table II, demonstrates that the relay with conventional approach calculates $R_{a p p}^{c o n v}$ as $96.71 \Omega$ and $X_{a p p}^{c o n v}$ as $-40.1 \Omega$, which lie outside the zone-1 of the relay (as shown in Fig. 13(a)). On the other hand, the relay using proposed approach calculates $R_{M F}^{\text {prop }}$ as $1.61 \Omega$ and $X_{M F}^{\text {prop }}$ as $23.03 \Omega$ and identifies the fault with actual faulted line section impedance. This confirms the improved performance of the proposed method in identifying different types of faults and also to be independent of fault resistance.

TABLE II

PERformance EVAluation for DifFEREnt Fault Types and Fault RESISTANCES AND COMPARATIVE ASSESSMENT

\begin{tabular}{|c|c|c|c|c|c|c|c|}
\hline \multirow{2}{*}{$\begin{array}{l}\text { Fault } \\
\text { Type }\end{array}$} & \multirow{2}{*}{$\begin{array}{l}R_{F} \\
(\Omega)\end{array}$} & \multicolumn{2}{|c|}{$Z_{M F}^{a c t}$} & \multicolumn{2}{|c|}{$Z_{a p p}^{c o n v}$} & \multicolumn{2}{|c|}{$Z_{M F}^{\text {prop }}$} \\
\hline & & $\begin{array}{c}\mathrm{R}_{\mathrm{MF}}^{\mathrm{act}} \\
(\Omega)\end{array}$ & $\begin{array}{c}\mathrm{X}_{\mathrm{MF}}^{\mathrm{act}} \\
(\Omega)\end{array}$ & $\begin{array}{c}\mathrm{R}_{\text {app }}^{\text {conv }} \\
(\Omega)\end{array}$ & $\begin{array}{c}\mathrm{X}_{\text {app }}^{\text {conv }} \\
(\Omega)\end{array}$ & $\begin{array}{c}\mathrm{R}_{\mathrm{MF}}^{\text {prop }} \\
(\Omega)\end{array}$ & $\begin{array}{c}\mathrm{X}_{\mathrm{MF}}^{\text {prop }} \\
(\Omega)\end{array}$ \\
\hline \multirow{4}{*}{ AG } & 5 & 1.6 & 22.94 & 6.49 & 18.20 & 1.59 & 22.87 \\
\hline & 30 & 1.6 & 22.94 & 36.20 & 11.05 & 1.60 & 22.99 \\
\hline & 50 & 1.6 & 22.94 & 53.85 & 7.49 & 1.60 & 22.95 \\
\hline & 100 & 1.6 & 22.94 & 84.03 & 1.72 & 1.60 & 22.96 \\
\hline \multirow{2}{*}{ BC } & 5 & 0.6 & 21.74 & 1.61 & 23.15 & 1.61 & 23.15 \\
\hline & 30 & 1.6 & 22.94 & 2.48 & -1.57 & 1.63 & 23.36 \\
\hline \multirow{4}{*}{ BCG } & 5 & 1.6 & 22.94 & -3.95 & 17.09 & 1.60 & 22.97 \\
\hline & 30 & 1.6 & 22.94 & 47.72 & -29.60 & 1.60 & 22.96 \\
\hline & 50 & 1.6 & 22.94 & 96.71 & -40.10 & 1.61 & 23.03 \\
\hline & 100 & 1.6 & 22.94 & 143.12 & -36.28 & 1.60 & 22.94 \\
\hline \multirow{2}{*}{$\mathrm{ABC}$} & 5 & 1.6 & 22.94 & 10.8 & 23.25 & 1.57 & 22.49 \\
\hline & 30 & 1.6 & 22.94 & 1.48 & 4.86 & 1.61 & 23.06 \\
\hline
\end{tabular}

\section{Performance evaluation for faults at different locations and Comparative assessment}

The severity of a fault depends on its location from the relay. Control algorithm associated with the interfacing converters acts in accordance with the fault severity. This results in variation in fault current characteristics for faults at different locations. It affects the apparent impedance seen by a conventional distance relay significantly. The result in Fig. 15 shows the failure of distance relay at bus 19 in identifying BCG faults at different locations in line 19-16 with a fault resistance of $40 \Omega$. The proposed adaptive relaying technique determines the deviation angle $\alpha$ by calculating the phase angle associated with the faulted loop current and thereby uses it to obtain the line impedance up to fault point for all cases accurately. A zone-2 fault (external to the protected line) is created in line 16-17 at a distance of $0.1 \mathrm{pu}$ from bus 16 . Homogeneity in conventional transmission network implies the $\mathrm{X} / \mathrm{R}$ ratio of all the lines in the network to be nearly equal. This allows the proposed method to calculate the impedance of the faulted line section as a multiple of the protected line impedance in case of external fault also. This is evident from the result shown in Fig. 15, whereas the conventional distance relay fails to find the fault in the concerned zone. This clearly shows the improved performance of the proposed method in identifying internal and external faults accurately with their actual locations.

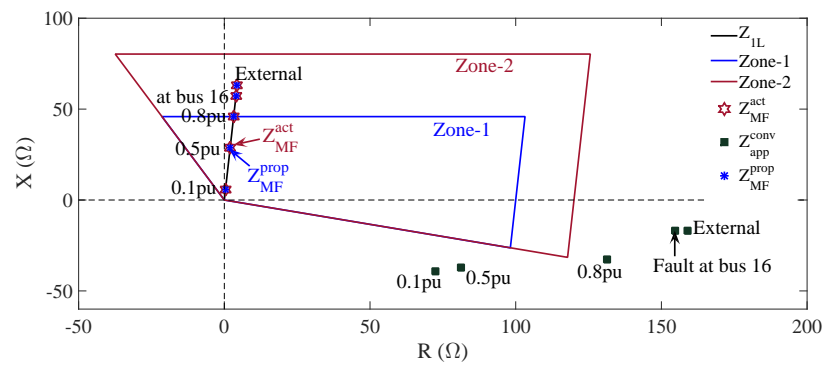

Fig. 15. Performance of the proposed and conventional methods for faults at different locations.

D. Performance evaluation considering uncertainty in renewable generation and variation in system condition

A large renewable plant consists of multiple numbers of units. At an instant, all units may not be operational due to maintenance or variability in weather condition resulting variation in output power. Distance protection becomes challenging in such a situation due to variable SIR condition. Weak grid condition introduces higher equivalent impedance of the grid $\left(Z_{1 G}\right)$. In such situation, the proposed method may result in an error due to the approximation considered in (18), especially for faults close to the remote bus. High value of pure-fault impedance of the solar plant keeps the error within a tolerable limit, which is demonstrated in this section. Results are provided in Table III for BCG faults in line 19-16 at a distance of $0.8 p u$ from bus 19 and with a fault resistance of $50 \Omega$. The value of $Z_{1 G}$ is varied up to 10 times of the actual value (by replacing the network connected at bus 16 with its Thevenin equivalent) with the solar plant generating at $100 \%$ and $50 \%$ of its rated output of $300 \mathrm{MW}$. Results show that the error decreases with the reduction in solar plant output, as it causes increment in pure-fault impedance of the solar plant. It is also evident from the results that the proposed method causes a maximum error of $2.92 \%$ with $Z_{1 G}$ of 10 times of its actual value and solar plant generating at its rated output, which is much below compared to the $20 \%$ margin provided in the proposed approach for instantaneous tripping. This demonstrates the robustness of the proposed method for different system conditions.

\section{E. Performance evaluation for different types of CIRP and Comparative assessment}

Control schemes and converter arrangements associated with CIRPs vary with the source types. Thus the modulation of fault current and voltage differs significantly with the types of CIRP. The proposed method calculates the deviation angle $(\alpha)$ by estimating the angle associated with the current in the faulted loop. This is determined by calculating equivalent purefault impedance of the CIRP at every measurement instant during fault, which is independent of the source types and associated control schemes and thereby the proposed method can identify the faults with its accurate location in the presence of any type of CIRP. This is demonstrated with the performance of relay at bus 19, for BCG faults in line 19-16 at a 
TABLE III

PERFORMANCE EVALUATION CONSIDERING UNCERTAINTY IN RENEWABLE GENERATION AND VARIATION IN SYSTEM CONDITION

\begin{tabular}{|c|c|c|c|c|c|c|}
\hline \multirow{2}{*}{$\begin{array}{c}\text { Plant } \\
\text { Output } \\
(\%)\end{array}$} & \multirow[b]{2}{*}{$\begin{array}{l}Z_{1 G} \\
(\%)\end{array}$} & \multicolumn{2}{|c|}{$Z_{M F}^{a c t}$} & \multicolumn{2}{|c|}{$Z_{M F}^{\text {prop }}$} & \multirow{2}{*}{$\begin{array}{c}\text { Error in } \\
\text { Calculated } \\
\text { Distance }\end{array}$} \\
\hline & & $\begin{array}{c}R_{M F}^{a c t} \\
(\Omega)\end{array}$ & $\begin{array}{c}X_{M F}^{a c t} \\
(\Omega)\end{array}$ & $\begin{array}{c}R_{M F}^{\text {prop }} \\
(\Omega)\end{array}$ & $\begin{array}{c}X_{M F}^{\text {prop }} \\
(\Omega)\end{array}$ & \\
\hline \multirow{4}{*}{100} & 100 & 3.2 & 45.9 & 3.20 & 45.89 & $0.03 \%$ \\
\hline & 200 & 3.2 & 45.9 & 3.21 & 45.96 & $0.19 \%$ \\
\hline & 500 & 3.2 & 45.9 & 3.24 & 46.46 & $1.29 \%$ \\
\hline & 1000 & 3.2 & 45.9 & 3.29 & 47.21 & $2.92 \%$ \\
\hline \multirow{4}{*}{50} & 100 & 3.2 & 45.9 & 3.20 & 45.91 & $0.09 \%$ \\
\hline & 200 & 3.2 & 45.9 & 3.19 & 45.79 & $0.18 \%$ \\
\hline & 500 & 3.2 & 45.9 & 3.24 & 46.43 & $1.21 \%$ \\
\hline & 1000 & 3.2 & 45.9 & 3.29 & 47.13 & $2.74 \%$ \\
\hline
\end{tabular}

distance of $0.4 p u$ from bus 19 with a fault resistance of $60 \Omega$. The solar plant connected at bus 33 is replaced by type-III and type-IV wind farms, one at a time. Results in Fig. 16 show that the conventional distance relay calculates different apparent impedances with variation in CIRP types and the impedances lie far away from the actual fault location. It is also observed that the relay cannot identify the fault within its zone-1 characteristic for all the cases. On the other hand, the proposed method identifies the faults in zone-1 correctly with its accurate location. This shows the adaptability of the proposed method with different types of CIRP.

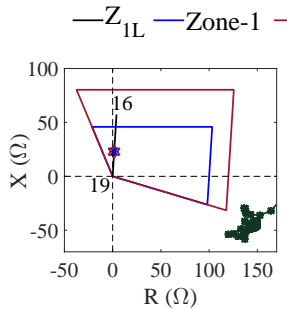

(a)

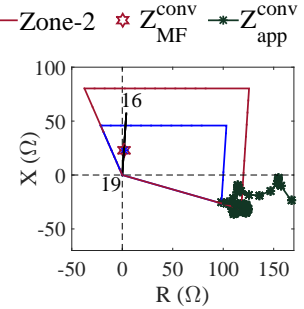

(b)

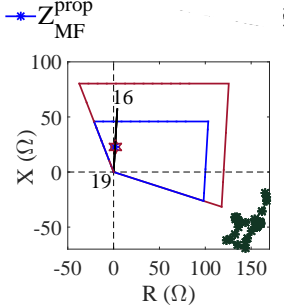

(c)
Fig. 16. Performance of the proposed method in the presence of (a) solar plant, (b) Type-III wind farm and (c) Type-IV wind farm.

\section{F. Performance evaluation for fault during power swing}

A distance relay finds challenges during power swing. Unintentional tripping may occur for a non-fault condition if the apparent impedance enters the protection zones during power swing. Correct operation for a fault during power swing is another aspect for distance protection. Performance of the proposed method is tested for such a situation.

A 300 MW Type-III wind farm is connected at bus 38 of the 39-bus New England system of Fig. 1, sharing the total generation of the existing synchronous generator. A three phase fault is created in line 26-29 at 7.3s, which is cleared by opening the circuit breakers at both ends of the line at $7.35 \mathrm{~s}$. As a result, a power swing is observed in the system. An AG fault is created in line 26-28 at $8 \mathrm{~s}$ with a fault resistance of $20 \Omega$. Fig. 17(a) and (b) show the voltage and current waveforms as seen by the relay at bus 28 protecting line 26-28. Following the detection of fault, the proposed method determines the phase angle of the faulted loop current by calculating equivalent pure-fault impedance of the source side for every measurement samples available to the relay and obtains the line impedance up to fault point. The resistance and reactance associated $Z_{M F}^{\text {prop }}$ obtained by the proposed approach following fault detection are shown in Fig. 17(c) and (d) with the $Z_{a p p}^{c o n v}$, calculated by the relay. Fig. 18 shows that $Z_{a p p}^{c o n v}$ calculated by the relay using conventional approach settles outside the relay characteristics resulting relay maloperation, whereas the proposed method obtains $Z_{M F}^{\text {prop }}$ identifying the fault point accurately. This demonstrates the superiority of the proposed method, even for a fault during power swing.
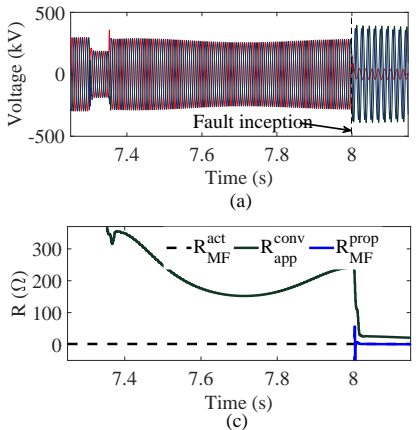

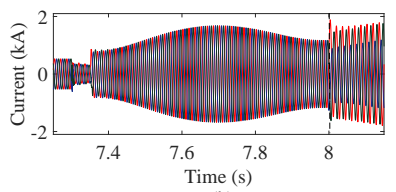

(b)

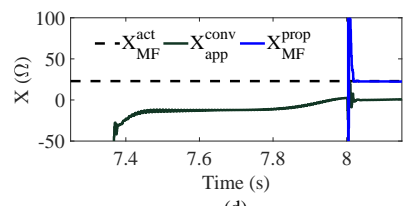

Fig. 17. Performance of proposed method for fault during power swing showing (a) voltage, (b) current seen by the relay, (c) resistance and (d) reactance of line impedance up to fault point.

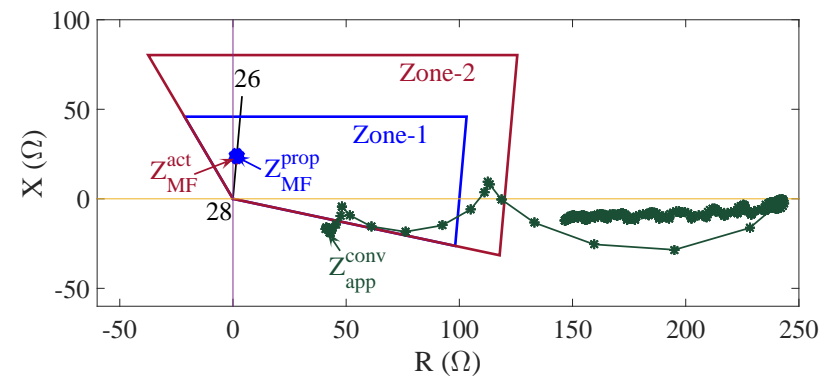

Fig. 18. Performance of proposed method for fault during power swing using distance relay characteristics.

\section{CONCLUSION}

The control operation of the converters and the variability associated with a renewable plant influence the fault characteristics of the network and affect the performance of the distance relay protecting the connected transmission line. Conventional distance relaying algorithm fails to identify the faults in prescribed zones at times, especially in case of faults with significant fault resistance. A distance protection approach adaptive to such fault characteristic variation is proposed for lines connecting CIRP. Proposed method uses local voltage and current data and calculates the line impedance up to the fault point from relay by determining the phase angle associated with the current in the faulted loop to obtain correct protection decision. The improved performance of the proposed adaptive method is demonstrated for different types of faults with variation in fault location, fault resistance, system condition including power swing and types of renewable plant. Comparative assessment with conventional distance relaying method shows the strength of the proposed method. 


\section{REFERENCES}

[1] "Guidelines for implementation of scheme for setting up of 750 MW grid-connected solar PV power projects under batch-1," Jawharlal Nehru National Solar Mission, Ministry of New and Renewable Energy, New Delhi, India, Tech. Rep., October 2013, [Online]. Available: http://mnre.gov.in/file-manager/UserFiles/finalVGF_750MW_Guidelines_for-grid-solar-power-projects.pdf.

[2] H. Lund and B. V. Mathiesen, "Energy system analysis of $100 \%$ renewable energy systemsthe case of denmark in years 2030 and 2050," Energy, vol. 34, no. 5, pp. 524-531, 2009.

[3] Z. Shuai, C. Shen, X. Yin, X. Liu, and J. Shen, "Fault analysis of inverter-interfaced distributed generators with different control schemes," IEEE Trans. Power Del., vol. PP, no. 99, pp. 1-1, 2017.

[4] H. M. Hasanien, "An adaptive control strategy for low voltage ride through capability enhancement of grid-connected photovoltaic power plants," IEEE Trans. Power Syst., vol. 31, no. 4, pp. 3230-3237, July 2016.

[5] Y. Wang and B. Ren, "Fault ride-through enhancement for grid-tied PV systems with robust control," IEEE Trans. Ind. Electr., vol. 65, no. 3, pp. 2302-2312, March 2018.

[6] K. Jia, Z. Yang, Y. Fang, T. Bi, and M. Sumner, "Influence of inverterinterfaced renewable energy generators on directional relay and an improved scheme," IEEE Trans. Power Electr., vol. 34, no. 12, pp. 11843-11855, Dec 2019.

[7] A. Banaiemoqadam, A. Hooshyar, and M. A. Azzouz, "A control-based solution for distance protection of lines connected to converter-interfaced sources during asymmetrical faults," IEEE Trans. Power Del., 2019, available in early access.

[8] A. Hooshyar, M. A. Azzouz, and E. F. El-Saadany, "Distance protection of lines emanating from full-scale converter-interfaced renewable energy power plants; part I: Problem statement," IEEE Trans. Power Del., vol. 30, no. 4, pp. 1770-1780, Aug 2015.

[9] Y. Fang, K. Jia, Z. Yang, Y. Li, and T. Bi, "Impact of inverter-interfaced renewable energy generators on distance protection and an improved scheme," IEEE Trans. Ind. Electr., vol. 66, no. 9, pp. 7078-7088, Sep. 2019.

[10] K. E. Arroudi and G. Joos, "Performance of interconnection protection based on distance relaying for wind power distributed generation." IEEE Trans. Power Del., vol. 33, no. 2, pp. 620-629, April 2018.

[11] A. K. Pradhan and G. Joos, "Adaptive distance relay setting for lines connecting wind farms," IEEE Trans. Energy Conv., vol. 22, no. 1, pp. 206-213, March 2007.

[12] A. Hooshyar, M. A. Azzouz, and E. F. El-Saadany, "Distance protection of lines connected to induction generator-based wind farms during balanced faults," IEEE Trans. Sustainable Energy, vol. 5, no. 4, pp. 1193-1203, Oct 2014.

[13] A. Hooshyar, M. A. Azzouz, and E. F. El-Saadany, "Distance protection of lines emanating from full-scale converter-interfaced renewable energy power plants ; part II: Solution description and evaluation," IEEE Trans. Power Del., vol. 30, no. 4, pp. 1781-1791, Aug 2015.

[14] Y. Liang, Z. Lu, W. Li, W. Zha, and Y. Huo, "A novel fault impedance calculation method for distance protection against fault resistance," IEEE Trans. Power Del., vol. 35, no. 1, pp. 396-407, Feb 2020.

[15] J. Ma, W. Ma, Y. Qiu, and J. Thorp, "An adaptive distance protection scheme based on the voltage drop equation," IEEE Trans. Power Del., vol. 30, no. 4, pp. 1931-1940, Aug 2015.

[16] V. Makwana and B. Bhalja, "A new digital distance relaying scheme for compensation of high-resistance faults on transmission line," IEEE Trans. Power Del., vol. 27, no. 4, pp. 2133-2140, Oct 2012.

[17] S. Paladhi and A. K. Pradhan, "Adaptive zone-1 setting following structural and operational changes in power system," IEEE Trans. Power Del., vol. 33, no. 2, pp. 560-569, April 2018.

[18] M. Thompson and A. Somani, "A tutorial on calculating source impedance ratios for determining line length," in Proc. 68th Annual Conference for Protective Relay Engineers, March 2015, pp. 833-841.

[19] G. E. Alexander, J. G. Andrichak, and W. Z. Tyska, "Relaying short lines," GE Power Management, Tech. Rep., [Online]. Available: http://store.gedigitalenergy.com/faq/documents/alps/ger-3735.pdf.

[20] Siemens, "Distance protection relay for transmission lines," Tech. Rep., 1999, [Online]. Available: ftp://ftp.socdu.ru/RZA/Siemens/SIPROTEC\%20SA522/7SA522_catalogue.pdf.

[21] Alstom, "Network protection and automation guide," Tech. Rep. 978-09568678-0-3, May 2011.

[22] CIGRE, "Modern distance protection functions and applications," WG B5.15, Tech. Rep., October 2008.
[23] I. Hiskens, "IEEE PES task force on benchmark systems for stability controls," Tech. Rep., November 2013, [Online]. Available: http://eioc.pnnl.gov/benchmark/ieeess/IEEE39/New_England_Reduced_ Model_(39_bus_system)_MATLAB_study_report.pdf.

[24] C. Loutan, P. Klauer, S. Chowdhury, S. Hall, M. Morjaria, V. Chadliev, N. Milam, C. Milan, and V. Gevorgian, "Demonstration of essential reliability services by a 300-MW solar photovoltaic power plant," National Renewable Energy Lab.(NREL), Golden, CO (United States), Tech. Rep., 2017.

[25] M. A. Azzouz, A. Hooshyar, and E. F. El-Saadany, "Resilience enhancement of microgrids with inverter-interfaced dgs by enabling faulty phase selection," IEEE Trans. Smart Grid, vol. 9, no. 6, pp. 6578-6589, 2018.

[26] A. Hooshyar, E. F. El-Saadany, and M. Sanaye-Pasand, "Fault type classification in microgrids including photovoltaic DGs," IEEE Trans. Smart Grid, vol. 7, no. 5, pp. 2218-2229, Sept 2016.

[27] E. Afshari, G. R. Moradi, Y. Yang, B. Farhangi, and S. Farhangi, "A review on current reference calculation of three-phase grid-connected pv converters under grid faults," in IEEE Power and Energy Conference at Illinois (PECI), 2017, pp. 1-7.

[28] PSCAD, "Type 3 wind turbine model," Tech. Rep., November 2018, [Online]. Available: https://www.pscad.com/uploads/knowledge_base/type_3_wind_turbine _model.pdf.

[29] PSCAD, "Type 4 wind turbine model," Tech. Rep., December 2018, [Online]. Available: https://www.pscad.com/uploads/knowledge_base/type_4_wind_turbine model_v46.pdf.

[30] NERC, "Reliability guideline: Improvements to interconnection requirements for BPS-connected inverter-based resources," Atlanta, GA 30326, Tech. Rep., September 2018.

[31] G. Ziegler, Numerical Distance Protection: Principles and Applications. John Wiley \& Sons, 2011.

[32] C. K. Alexander, Fundamentals of Electric Circuits. McGraw-Hill, 2009.

[33] S. Jingliao, L. Yongli, L. Shengwei, and J. Qiang, "Study on adaptive current instantaneous trip protection scheme for distribution network with inverter interfaced DG," Automation of Electric Power Systems, vol. 14, p. 017, 2009.

[34] K. Jia, Z. Yang, Z. Zhu, Y. Fang, Q. Zhao, and B. Liu, "Current amplitude ratio based pilot protection for the transmission line connected to inverter-interfaced renewable energy power plants," in IEEE Innovative Smart Grid Technologies - Asia (ISGT Asia), May 2019, pp. 2090-2094.

[35] G. Kou, L. Chen, P. VanSant, F. Velez-Cedeno, and Y. Liu, "Fault characteristics of distributed solar generation," IEEE Trans. Power Del., vol. 35, no. 2, pp. 1062-1064, April 2020.

[36] A. Haddadi, I. Kocar, and E. Farantatos, "Impact of inverter-based resources on protection schemes based on negative sequence components," EPRI, Technical Brief - Bulk Power System Integration of Variable Generation, July 2019.

[37] S. H. Horowitz and A. G. Phadke, Power System Relaying. John Wiley \& Sons, 2008, pp. 108-109.

\section{BIOGRAPHIES}

Subhadeep Paladhi (S'14) is currently working toward the Ph.D. degree in Electrical Engineering at the Indian Institute of Technology, Kharagpur, India. His current research interest is power system protection.

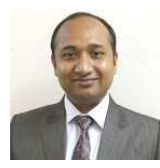

Ashok Kumar Pradhan (SM'10, M'94) is a Professor in the Department of Electrical Engineering, Indian Institute of Technology, Kharagpur, India. His research interests include power system relaying and monitoring. 